\title{
Efeitos cardiovasculares da anestesia dissociativa na reposição volêmica com colóide e solução hipertônica em cães: avaliação biotelemétrica
}

\author{
[Cardiovascular effects of dissociative anesthesia in the volemic expansion with colloid and hypertonic \\ solution in dogs: biotelemetric evaluation] \\ N. Oleskovicz ${ }^{1}$, J.C.D. Moreno ${ }^{2}$, E.C.P. Guirro ${ }^{3}$, C.A.A. Valadão ${ }^{4}$, D.T. Fantoni ${ }^{5}$ \\ ${ }^{1}$ Hospital de Clínica Veterinária - UDESC \\ Avenida Luiz de Camões, 2090 \\ 88520-000 - Lages, SC \\ ${ }^{2}$ Universidade de Franca - Franca, SP \\ ${ }^{3}$ Universidade Federal do Paraná - Câmpus Palotina - Palotina, PR \\ ${ }^{4}$ Faculdade de Ciências Agrárias e Veterinárias - UNESP - Jaboticabal, SP \\ ${ }^{5}$ Faculdade de Medicina Veterinária e Zzootecnia - USP - São Paulo, SP
}

\begin{abstract}
RESUMO
Avaliaram-se os efeitos cardiovasculares por um período de 24 horas, após a administração de solução salina hipertônica $(\mathrm{NaCl}) 7,5 \%$ ou em associação ao hidroxietilamido 130/0,4 (HES), em cães com hipovolemia induzida e tratados com cetamina levógira ou racêmica. Após a indução da hipovolemia, administrou-se $\mathrm{NaCl}$ $7,5 \%(4 \mathrm{~mL} / \mathrm{kg})$ no grupo hipertônica levógira (GHL) e no grupo hipertônica racêmica (GHR) ou HES 130/0,4 na mesma proporção de sangue retirado, associado a $\mathrm{NaCl} 7,5 \%(4 \mathrm{~mL} / \mathrm{kg})$ no grupo hipertônica colóide levógira (GHCL) e no grupo hipertônica colóide racêmica (GHCR). Após 30 minutos, administrou-se por via intravenosa, cetamina levógira (CL; $5 \mathrm{mg} / \mathrm{kg}$ ) no GHL e GHCL ou cetamina racêmica (CR; $10 \mathrm{mg} / \mathrm{kg}$ ) no GHR e GHCR. A frequência cardíaca (FC) e a pressão arterial sistólica (PAS) foram menores após a hipovolemia e após a CR. A pressão arterial média (PAM) e a pressão arterial diastólica (PAD) foram menores após a hipovolemia e após a administração de CL e CR. Não foram observadas diferenças significativas entre os grupos em relação à FC, PAS, PAM e PAD durante o período de mensuração por biotelemetria desde T210 até T1440. A administração de HES associado ao $\mathrm{NaCl} 7,5 \%$ propiciou restabelecimento imediato da PAM, a administração de $\mathrm{NaCl} 7,5 \%$ não restaurou a PAM em pacientes hipovolêmicos, a administração de CR ou CL produziu efeitos semelhantes e todos os tratamentos mantiveram estáveis as pressões arteriais e a FC por um período de até 24 horas.
\end{abstract}

Palavras-chave: cão, solução hipertônica, hidroxietilamido, hipovolemia, biotelemtria

\begin{abstract}
The cardiovascular effects were evaluated for a 24-hour period, after the administration of hypertonic solution ( $\mathrm{NaCl} 7.5 \%)$ or in association with hidroxyethyl starch 130/0.4 (HES) in dogs under induced experimental hypovolemia and treated with racemic ketamine (RK) or S(+) ketamine (SK). After the hypovolemia induction, administration of $\mathrm{NaCl} 7.5 \%\left(4 \mathrm{~mL} \cdot \mathrm{kg}^{-1}\right)$ was performed in two groups named hypertonic $S(+)$ (HSG) and hypertonic racemic (HRG), or $\mathrm{NaCl} 7.5 \%\left(4 \mathrm{~mL} . \mathrm{kg}^{-1}\right)$ in association with $\mathrm{HES}$, in the same ratio of removed blood, in two groups named hypertonic colloid $S(+)$ (HCSG) and hypertonic colloid racemic (HCRG). After 30 minutes, SK (5mg. $\left.\mathrm{kg}^{-1}\right)$ was administered by intravenous injection in HSG and HCSG groups, or RK $\left(10 \mathrm{mg} . \mathrm{kg}^{-1}\right)$ in HRG and HCRG groups. The heart rate (HR) and systolic arterial pressure (SAP) were lower after hypovolemia and RK. Mean (MAP) and diastolic arterial pressure (DAP) were reduced after hypovolemia and either SK or RK administration. Significant differences were not observed among the groups to HR, SAP, $M A P$, and DAP during the biotelemetry mensuration period, from T210 to T1440. HES associated with $\mathrm{NaCl}$ $7.5 \%$ administration propitiated immediate re-establishment of $\mathrm{MAP} . \mathrm{NaCl} 7.5 \%$ administration did not restore MAP in hypovolemic patients. Either RK or SK administrations produced similar effects and all of the treatments maintained stable blood pressure and heart rate for a 24-hour period.
\end{abstract}

Keywords: dog, hypertonic solution, hidroxyethylstarch, hypovolemia, biotelemetry

Recebido em 10 de março de 2008

Aceito em 23 de outubro de 2008

E-mail: a2no@cav.udesc.br 


\section{INTRODUÇÃO}

O choque hemorrágico é caracterizado por redução do volume sanguíneo, o qual diminui o transporte de oxigênio e a perfusão tecidual. Frequentemente, pacientes com quadros de hipovolemia de grau moderado a severo são submetidos a intervenções cirúrgicas emergenciais, para identificar e corrigir as possíveis lesões, visando preservar a vida do paciente (Ingwersen et al., 1988).

As soluções cristaloides isotônicas foram muito utilizadas, como principal fluido para reposição volêmica. No entanto, muitos estudos demonstram resultados apenas satisfatórios com o uso destas substâncias, sobretudo no tratamento do choque hemorrágico grave. A constatação de que o emprego de pequeno volume de solução hipertônica de cloreto de sódio $(\mathrm{NaCl} 7,5 \%)$ promovia restabelecimento imediato e definitivo dos parâmetros hemodinâmicos de cães submetidos a choque hemorrágico grave fez surgir um novo conceito na terapia do choque. A administração de solução hipertônica aumentou a pressão arterial média (PAM), o débito cardíaco (DC) e corrigiu os distúrbios metabólicos, por período superior a seis horas (Velasco et al.,1980; Kramer, 1986). A taxa de sobrevida foi de $100 \%$ nos animais tratados com $\mathrm{NaCl} 7,5 \%$ enquanto os animais que receberam somente $\mathrm{NaCl} 0,9 \%$ morreram nas primeiras 6,5 horas após a indução da hipovolemia.

Os coloides têm sido efetivos em pequeno volume e promovem expansão volêmica de longa duração, devido à elevada pressão oncótica. $\mathrm{O}$ hidroxietilamido 130/0,4 (HES), obtido da amilopectina extraída do milho, apresenta alto peso molecular $(130 \mathrm{kDa})$ e grau de substituição molar de 0,4. Possui uma capacidade de expansão de aproximadamente $100 \%$, ou seja, aumenta o volume plasmático na mesma proporção do volume infundido por quatro a seis horas, o qual é comparável aos demais HES utilizados (Konrad et al., 2000).

O choque hemorrágico produz grave comprometimento hemodinâmico e representa um desafio, principalmente nos casos clínicos em que o paciente necessita ser anestesiado. Haskins et al. (2005) observaram os efeitos cardiopulmonares em cães submetidos ao choque hemorrágico e constataram aumento na frequência cardíaca (FC), resistência vascular sistêmica (RVS) e pulmonar e frequência respiratória (FR), bem como diminuição da PAM, pressão venosa central (PVC), DC e transporte de oxigênio. A indução anestésica em pacientes hipovolêmicos é a principal causa de mortalidade, provavelmente devido ao fato de os fármacos utilizados interferirem nos reflexos cardiovasculares e nas respostas do sistema nervoso simpático (Ingwersen et al., 1988). Neste sentido, com relação à utilização da cetamina levógira, são escassas as informações referentes aos efeitos cardiovasculares quando usada na indução ou manutenção anestésica em cães submetidos ao choque hemorrágico.

A monitoração prolongada desses pacientes é de suma importância, visto que as substâncias utilizadas para expansão volêmica produzem efeito hemodinâmico por períodos relativamente curtos. Várias técnicas têm sido empregadas para determinar as alterações clínicas, entretanto, a manipulação para a colheita dos dados produz interferência nos dados dependentes de ativação humoral, como a FC e a pressão arterial (PA). Neste contexto destaca-se a biotelemetria, que possibilita monitorar vários animais ao mesmo tempo, mensurando diferentes parâmetros fisiológicos por longos períodos sem interferência no padrão de comportamento (Truett e West, 1995). Os altos níveis de confiabilidade e fidelidade dos dados obtidos pela biotelemetria, em relação aos obtidos por mensuração direta, permitiram o uso desta técnica em estudos sobre os mecanismos de ação dos barorreceptores em condições fisiológicas e durante o sono (Anderson, 1999).

Neste estudo, objetivou-se avaliar a frequência cardíaca e a pressão arterial por um período de até 24 horas, por meio da avaliação biotelemétrica, após a reposição volêmica com o uso isolado de solução salina hipertônica 7,5\% ou em associação ao hidroxietilamido 130/0,4, em cães com hipovolemia induzida, submetidos à anestesia com cetamina levógira ou cetamina racêmica.

\section{MATERIAL E MÉTODOS}

Este estudo foi aprovado pela Comissão de Ética e do Bem Estar dos Animais (CEBEA) do Campus de Jaboticabal da UNESP, conforme 
protocolo $\mathrm{n}^{\mathrm{o}}$ 005750. Foram utilizadas quatro cadelas Beagles, pesando entre 8 e $10 \mathrm{~kg}$, com 10 a 12 meses de idade, mantidas em gaiolas metabólicas individuais, diariamente manejadas e condicionadas ao ambiente de experimentação durante seis meses. Após a fase de adaptação, os animais submetidos à ovariossalpingohisterectomia (OSH) e esplenectomia foram submetidos ao protocolo experimental quatro vezes, com intervalos de 35 dias, seguindo a metodologia do quadrado latino. Realizaram-se exames clínicos e laboratoriais periódicos ao início de cada etapa experimental.

Para implantação cirúrgica dos transmissores, as cadelas receberam medicação pré-anestésica (MPA) com cloridrato de levomepromazina ${ }^{1}$ na dose de $1 \mathrm{mg} / \mathrm{kg}$ e buprenorfina ${ }^{2}$ na dose de $0,01 \mathrm{mg} / \mathrm{kg}$, na mesma seringa, por via IM. Após 25 minutos, administrou-se propofol ${ }^{3}$ na dose de $5 \mathrm{mg} / \mathrm{kg}$ por via IV. Na sequência, os animais foram intubados com sonda de Magill de diâmetro adequado e mantidos sob anestesia geral inalatória, em sistema com reinalação parcial de gases, com sevoflurano ${ }^{4}$ (1,5 CAM) diluído em oxigênio $100 \%$, com fluxo de $30 \mathrm{~mL} / \mathrm{kg}$, a qual foi estabelecida pela leitura da concentração da mistura anestésica expirada ${ }^{5}$.

Após a preparação do campo operatório, realizou-se incisão para-sagital de $10 \mathrm{~cm}$ no flanco esquerdo, divulsionando-se a tela subcutânea permitindo a implantação da unidade de transmissão (Fig. 1A). Subsequentemente, outra incisão de $3 \mathrm{~cm}$ foi realizada na face medial do membro pélvico esquerdo, sobre o plexo femoral, isolando-se a artéria femoral caudal, procedendo-se à introdução e à fixação do cateter em seu lúmen (Fig. 1B, 1C, 1D).

Adicionalmente, um dos eletrodos de ECG foi direcionado sob a pele e implantado na região axilar direita. $\mathrm{O}$ outro foi posicionado na região abdominal ventral esquerda. Ato contínuo procedeu-se à síntese do tecido subcutâneo e pele. A antibioticoterapia pós-operatória

\footnotetext{
${ }^{1}$ Neozine, Aventis $25 \mathrm{mg} / 5 \mathrm{~mL}$ - São Paulo, Brasil.

${ }^{2}$ Temgesic, Schering Plough S.A. - São Paulo, Brasil.

${ }^{3}$ Propoabbott, Abbott - São Paulo, Brasil.

${ }^{4}$ Sevocris, Cristália Produtos Químicos e Farmacêuticos Ltda - Itapira, Brasil.

${ }^{5}$ OHMEDA- RGM 5250 - Datex Ohmeda - Miami, EUA

(Proc. Fapesp 96/12830-0).
}

consistiu da aplicação diária de enrofloxacina ${ }^{6}$ na dose de $5 \mathrm{mg} / \mathrm{kg} \mathrm{SC}$, durante sete dias, meloxicam ${ }^{7}$ na dose de $0,2 \mathrm{mg} / \mathrm{kg}$ no primeiro dia e $0,1 \mathrm{mg} / \mathrm{kg}$ nos três dias subsequentes, $\mathrm{SC}$ e buprenorfina $^{2}$ na dose de $0,01 \mathrm{mg} / \mathrm{kg}, \mathrm{SC}$, durante quatro dias, os animais foram mantidos com colar elizabetano durante sete dias.

Decorridos 30 dias da implantação dos transmissores, as cadelas foram colocadas em decúbito lateral direito, sobre colchão térmico ativo e anestesiadas com sevoflurano administrado por máscara facial com fluxo diluente de oxigênio de $41 / \mathrm{min}$. Após intubação orotraqueal com sonda de Magill, a manutenção anestésica foi realizada com sevoflurano 1 CAM (aferida pela leitura no analisador de gases anestésicos ${ }^{8}$ ) diluído em oxigênio a $100 \%$, com fluxo de $30 \mathrm{~mL} / \mathrm{kg}$, administrados por meio de circuito anestésico com reinalação parcial de gases, dotado de vaporizador calibrado para o agente anestésico utilizado.

Subsequentemente, foi dissecada a artéria tibial dorsal para a introdução de um cateter de polietileno $(22 \mathrm{G})$, utilizado para mensuração das pressões arteriais. Após o preparo, os animais foram mantidos sob anestesia geral inalatória com sevofluorano 1 CAM durante 30 minutos. Depois de estabilizado o plano anestésico, os animais continuaram sob anestesia geral inalatória até o final do período de avaliação direta, 180 minutos (T180).

Após 30 minutos de estabilização da anestesia, retirou-se um volume não pré-determinado de sangue, por meio da artéria tibial dorsal, com taxa máxima de extração de $20 \mathrm{~mL} / \mathrm{min}$, até atingir valores de PAM entre 45 e $55 \mathrm{mmHg}$. A pressão foi mantida nessa faixa durante 30 minutos, seja pela extração ou pela infusão de alíquotas adicionais de sangue, se necessário. $\mathrm{O}$ sangue extraído foi armazenado em bolsas de transfusão sanguínea e mantido sob refrigeração, para ser reinfundido nos animais ao término de cada período de experimentação, imediatamente após o término da avaliação das 20 horas por biotelemetria, aproximadamente 24 horas após sua extração.

\footnotetext{
${ }^{6}$ Baytril, Bayer S.A - Saúde Animal - São Paulo, Brasil.

${ }^{7}$ Movatec, Boehringer Ingelheim - São Paulo, Brasil.

${ }^{8}$ Ohmeda - Mod. Excel 210 SE. - Miami, EUA. (Proc. Fapesp 97/10668-4).
} 


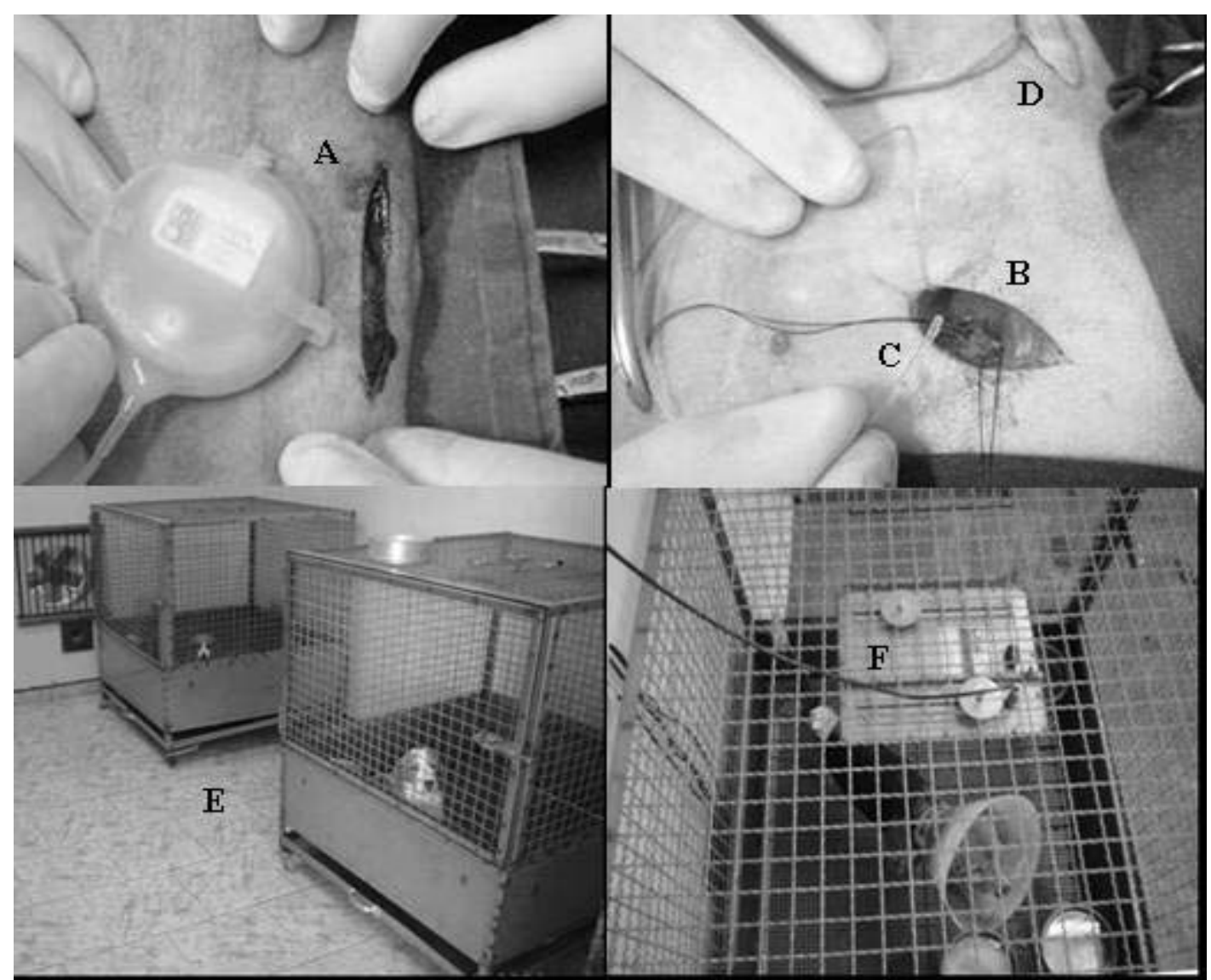

Figura 1. Implantação da unidade de transmissão, no espaço subcutâneo (A); dissecção cirúrgica da artéria femoral esquerda (B); cateter a ser implantado na artéria femoral (C); posicionamento transmissor (D); gaiolas individuais com receptores de sinais (E); vista superior da gaiola com antena receptora de sinais $(\mathrm{F})$.

Subsequentemente à indução da hipovolemia, administraram-se, injeções de $\mathrm{NaCl} 7,5 \%{ }^{9}$ na dose de $4 \mathrm{~mL} / \mathrm{kg}$ no grupo hipertônica levógira (GHL, n=4) e no grupo hipertônica racêmica (GHR, n=4) ou solução de hidroxietilamido (HES $130 / 0,4)^{10}$ na proporção de 1:1, em relação ao volume de sangue retirado de cada animal, associada a $\mathrm{NaCl} 7,5 \%$ na dose de $4 \mathrm{~mL} / \mathrm{kg}$ no grupo hipertônica coloide levógira $(\mathrm{GHCL}, \mathrm{n}=4)$ e no grupo hipertônica coloide racêmica (GHCR, $\mathrm{n}=4)$. Esse período, denominado de expansão, teve a duração total de 20 minutos para infusão das soluções, pela via intravenosa.

Após 30 minutos da fase de expansão, foi administrado por via intravenosa, em ensaio cego, o isômero $\mathrm{S}(+)$ da cetamina (cetamina

${ }^{9}$ Solução hipertônica de cloreto de sódio a 7,5 \%, Áster Prod. Médicos Ltda. - Sorocaba, Brasil.

${ }^{10}$ Voluven 6\%, $(130 / 0,4)$ Fresenius-Kabi - Campinas, Brasil. levógira $\left.{ }^{11}\right)(5 \mathrm{mg} / \mathrm{kg})$ no GHL e GHCL ou a cetamina racêmica ${ }^{12}(10 \mathrm{mg} / \mathrm{kg})$ no GHR e GHCR.

Em todos os grupos, foi administrada fluidoterapia de manutenção com solução salina fisiológica $0,9 \%$ na dose de $5 \mathrm{~mL} / \mathrm{kg}$ hora, iniciando-se 30 minutos após a hipovolemia (T0) por até 180 minutos (T180), totalizando três horas de fluidoterapia. Independente do grupo, após a administração do agente dissociativo, foi administrado solução de Ringer com lactato de sódio, durante o período de 120 minutos, em volume suficiente para estabilizar a pressão arterial média em $60 \mathrm{mmHg}$.

\footnotetext{
${ }^{11}$ Ketamin S(+), 50mg/mL, Cristália Produtos Químicos e Farmacêuticos Ltda. - Itapira, Brasil.

${ }^{12}$ Ketamin, 50mg/mL, Cristália Produtos Químicos e

Farmacêuticos Ltda. - Itapira, Brasil.
} 
Foram mensurados: frequência cardíaca (FC), por meio de eletrocardiógrafo computadorizado $^{13}$; pressão arterial sistólica (PAS), diastólica (PAD) e média (PAM), por meio de leitura no monitor multiparamétrico ${ }^{14}$ cujo transdutor foi conectado ao cateter introduzido na artéria tibial dorsal. Esses parâmetros foram mensurados nos seguintes tempos: 30 minutos após a instrumentação (T30), 30 minutos após a indução da hipovolemia (T0), imediatamente após a fase de expansão (T30), 15 e 30 minutos após a fase de expansão (T45 e T60), 15, 30, 45, 60, 90 e 120 minutos após a aplicação da cetamina levógira ou cetamina racêmica (T75, T90, T105, T120, T150 e T180).

Após o T180, foram mensurados por biotelemetria $^{15}$ os valores de PAS, PAM, PAD e FC, realizados de forma contínua pela matriz de consolidação. Neste estudo, optou-se por registro de intervalos de 10 minutos a partir do T210 (duas horas e meia após a aplicação da cetamina levógira ou cetamina racêmica) até o T1440 (24 horas) e, para efeito de comparação estatística entre os tempos e entre os grupos, optou-se pela utilização dos dados a cada 30 minutos, totalizando 41 aferições para cada grupo (Fig. 1E, 1F).

Os dados coletados foram submetidos à análise de variância de uma via com repetições múltiplas, seguidas da comparação pelo teste SNK $(\mathrm{P} \leq 0,05)$, para determinação das diferenças estatísticas entre médias ao longo do tempo dentro de cada grupo e médias em cada tempo entre os grupos.

\section{RESULTADOS E DISCUSSÃO}

Após a administração da cetamina, a quantidade de solução de Ringer com lactato de sódio administrado para manter a $\mathrm{PAM} \geq 60 \mathrm{mmHg}$ foi significativamente maior no GHR $(437,50 \pm 51,53 \mathrm{~mL})$, quando comparado ao GHL $(100,00 \pm 70,71 \mathrm{~mL})$, GHCL $(175,00 \pm 112,73 \mathrm{~mL})$ e GHCR $(137,50 \pm 137,50 \mathrm{~mL})$. Tan et al. (2002)

\footnotetext{
${ }^{13}$ TEB - mod. ECGPC software versão 1.10 - São Paulo, Brasil. (Proc. Fapesp 96/1151-5).

${ }^{14}$ Dixtal DX2010, Módulo Pressão Arterial Invasiva Manaus, Brasil. (Proc. Fapesp 96/02877-0).

${ }^{15}$ Data Sciences Inc. - St. Paul, EUA. (Proc. Fapesp 01/03304-3).
}

afirmaram que a solução $\mathrm{NaCl} \quad 7,5 \%$ em associação com outros expansores plasmáticos reduz o volume necessário para restaurar a PAM e o DC. Kreimeier et al. (1997) demonstraram que em casos de choque hemorrágico e traumático, a solução $\mathrm{NaCl} 7,5 \%$ isolada ou associada a coloides restabelece a PA e o DC, mantendo a resistência vascular periférica reduzida.

Em relação à FC, observou-se redução significativa nos valores médios após a indução da hipovolemia no GHL, GHCL e GHCR, bem como após administração da cetamina no GHCR. Cabe ressaltar que, mesmo havendo redução significativa, os valores mantiveram-se dentro da faixa fisiológica para a espécie. Konsayreeepong et al. (1993) afirmaram que a ação final da cetamina sobre o miocárdio permanece controversa, pois têm sido reportados tanto efeitos inotrópicos positivos como depressores do miocárdio, in vivo e in vitro. Cook et al. (1991) descreveram que em condições fisiológicas, quando o sistema simpático encontra-se íntegro, os efeitos estimulantes decorrentes da inibição da recaptação das catecolaminas predominam. Segundo Cromhout (2003), a cetamina, quando administrada em pacientes normovolêmicos, aumenta a $\mathrm{FC}$ em torno de $20 \%$, a PAS em torno de $25 \mathrm{mmHg}$ e o DC por dois a quatro minutos. Esses achados discordam dos resultados de Nam (1977), pois a administração de cetamina em cães submetidos à hipovolemia experimental, sem efeito de nenhum outro agente anestésico, produziu elevação da FC, sem alterações na FR.

Observou-se redução da PAS 15 minutos após da administração de cetamina em todos os grupos, sendo que, durante toda a fase de expansão (T30 até T60), essa variável encontrava-se próxima dos valores basais. Esses resultados assemelhamse aos descritos por Duque et al. (2005), que não observaram o aparecimento dos efeitos cardioestimulantes da cetamina, quando essa foi administrada em infusão contínua $(100 \mu \mathrm{g} / \mathrm{kg} / \mathrm{min})$ em cães hipovolêmicos anestesiados com desfluorano.

Para a PAM foram obtidos resultados semelhantes, no entanto para os grupos que receberam somente solução de $\mathrm{NaCl} 7,5 \%$ a PAM reduziu desde a fase de indução de hipovolemia até os 180 minutos (T180), sendo 
que, após a administração de cetamina, não foi observado agravamento do quadro (Tab. 1). Ocorreu redução da PAM, aos 15 e 30 minutos da administração de cetamina no GHCL e dos 15 até 45 minutos no GHCR. Houve diminuição significativa da PAD após a indução da hipovolemia em todos os grupos. Diminuição do T0 até três horas (T180) no GHL e GHR, diminuição após a administração de cetamina no GHCL e GHCR, quando comparado ao T-30. Não foram observadas diferenças significativas para os valores de FC, PAS, PAM e PAD do T210 até T1440. Especula-se que a adição dos efeitos da cetamina aos da anestesia pelo sevoflurano possa ter sido a responsável pela queda da PAM em ambos os grupos, apresentando efeito mais duradouro para cetamina racêmica do que para cetamina levógira, pois sabe-se que a infusão de agentes dissociativos reduz a concentração alveolar mínima dos agentes inalatórios deprimindo a atividade do centro vasomotor. De fato, isso pode ter ocorrido, pois, segundo Haskins et al. (2005), a administração de cetamina em cães com hipovolemia aumenta o DC, PA e FC.

Tabela 1. Médias e desvios-padrão da pressão arterial média ( $\mathrm{mmHg}$ ), em cadelas submetidas à hipovolemia experimental e tratadas com $\mathrm{NaCl} 7,5 \%$ associada ou não ao hidroxietilamido $(130 / 0,4)$ após administração de cetamina levógira ou cetamina racêmica

\begin{tabular}{|c|c|c|c|c|c|c|c|c|c|c|c|}
\hline & $\mathrm{T}-30$ & T0 & T30 & T45 & T60 & $\mathrm{T} 75$ & T90 & T105 & T120 & $\mathrm{T} 150$ & T180 \\
\hline \multirow[t]{2}{*}{ GHL } & 100 & $51^{*}$ & $80^{*}$ & $82 *$ & $67^{*}$ & $57^{*}$ & $67^{*}$ & $72 *$ & $69^{*}$ & $73^{*}$ & $70 *$ \\
\hline & \pm 6 & \pm 1 & \pm 8 & \pm 4 & \pm 4 & \pm 3 & \pm 3 & \pm 2 & \pm 3 & \pm 6 & \pm 4 \\
\hline \multirow[t]{2}{*}{ GHR } & 86 & $53 *$ & $73 *$ & $68^{*}$ & $60 *$ & $43 *$ & $62 *$ & $62 *$ & $66^{*}$ & $68 *$ & 76 \\
\hline & \pm 6 & \pm 3 & \pm 7 & \pm 8 & \pm 6 & \pm 2 & \pm 3 & \pm 2 & \pm 5 & \pm 6 & \pm 3 \\
\hline \multirow[t]{2}{*}{ GHCL } & 92 & $54^{*}$ & 74 & 79 & 76 & $66^{*}$ & $60 *$ & 71 & 76 & 80 & 80 \\
\hline & \pm 6 & \pm 2 & \pm 3 & \pm 7 & \pm 8 & \pm 9 & \pm 6 & \pm 4 & \pm 5 & \pm 4 & \pm 4 \\
\hline \multirow[t]{3}{*}{ GHCR } & 87 & $55^{*}$ & 74 & 76 & 71 & $52 *$ & $60 *$ & $62 *$ & 71 & 77 & 70 \\
\hline & \pm 4 & \pm 4 & \pm 8 & \pm 7 & \pm 6 & \pm 6 & \pm 6 & \pm 5 & \pm 4 & \pm 5 & \pm 10 \\
\hline & $\mathrm{T} 210$ & T240 & T300 & T360 & T420 & T480 & T540 & T600 & T660 & T720 & T780 \\
\hline \multirow[t]{2}{*}{ GHL } & 83 & 84 & 81 & 76 & 84 & 82 & 76 & 87 & 74 & 66 & 76 \\
\hline & \pm 10 & \pm 6 & \pm 7 & \pm 11 & \pm 10 & \pm 7 & \pm 8 & \pm 7 & \pm 8 & \pm 8 & \pm 9 \\
\hline \multirow[t]{2}{*}{ GHR } & 76 & 88 & 91 & 82 & 86 & 75 & 82 & 67 & 72 & 71 & 76 \\
\hline & \pm 7 & \pm 11 & \pm 15 & \pm 13 & \pm 9 & \pm 14 & \pm 11 & \pm 14 & \pm 10 & \pm 10 & \pm 9 \\
\hline \multirow[t]{2}{*}{ GHCL } & 97 & 86 & 101 & 88 & 82 & 76 & 85 & 85 & 78 & 79 & 91 \\
\hline & \pm 10 & \pm 11 & \pm 9 & \pm 3 & \pm 11 & \pm 7 & \pm 11 & \pm 8 & \pm 13 & \pm 8 & \pm 8 \\
\hline \multirow[t]{3}{*}{ GHCR } & 92 & 80 & 81 & 84 & 79 & 83 & 81 & 74 & 76 & 78 & 89 \\
\hline & \pm 7 & \pm 5 & \pm 5 & \pm 5 & \pm 9 & \pm 6 & \pm 7 & \pm 7 & \pm 4 & \pm 5 & \pm 5 \\
\hline & T840 & T900 & T960 & T1020 & T1080 & T1140 & T1200 & T1260 & T1310 & T1380 & T1440 \\
\hline \multirow[t]{2}{*}{ GHL } & 76 & 73 & 79 & 82 & 82 & 81 & 83 & 73 & 79 & 79 & 80 \\
\hline & \pm 10 & \pm 8 & \pm 2 & \pm 8 & \pm 4 & \pm 9 & \pm 5 & \pm 6 & \pm 7 & \pm 7 & \pm 7 \\
\hline \multirow[t]{2}{*}{ GHR } & 69 & 78 & 77 & 84 & 77 & 73 & 78 & 71 & 81 & 74 & 74 \\
\hline & \pm 12 & \pm 10 & \pm 9 & \pm 7 & \pm 10 & \pm 9 & \pm 6 & \pm 7 & \pm 5 & \pm 5 & \pm 9 \\
\hline \multirow[t]{2}{*}{ GHCL } & 81 & 89 & 82 & 83 & 89 & 83 & 91 & 77 & 93 & 85 & 86 \\
\hline & \pm 9 & \pm 6 & \pm 5 & \pm 11 & \pm 8 & \pm 12 & \pm 7 & \pm 10 & \pm 7 & \pm 9 & \pm 11 \\
\hline \multirow[t]{2}{*}{ GHCR } & 80 & 80 & 78 & 82 & 101 & 80 & 77 & 70 & 81 & 70 & 81 \\
\hline & \pm 7 & \pm 6 & \pm 8 & \pm 11 & \pm 10 & \pm 8 & \pm 4 & \pm 4 & \pm 7 & \pm 3 & \pm 6 \\
\hline
\end{tabular}

GHL: grupo hipertônica cetamina levógira, GHR: grupo hipertônica cetamina racêmica, GHCL: grupo hipertônica coloide cetamina levógira, GHCR: grupo hipertônica coloide cetamina racêmica. *Significativamente diferente de T30 , teste $\mathrm{SNK}(\mathrm{P} \leq 0,05)$.

T-30; 30 minutos após a instrumentação; T0 30 minutos após a indução da hipovolemia; T30: imediatamente após a fase de expansão; T45 e T60: 15 e 30 minutos após a fase de expansão; T75 a T180: 15, 30, 45, 60, 90 e 120 minutos após a aplicação de cetamina levógira ou racêmica; T210 a T1440: mensuração por biotelemetria a cada 10 minutos.

A supressão da resposta simpática observada neste estudo pode ainda ter sido causada pela interferência do agente inalatório, pois os animais permaneceram anestesiados com sevoflurano a 1 CAM, durante aproximadamente quatro horas, considerarando-se o período de instrumentação (30 minutos), de estabilização da anestesia (30 minutos) e os 180 minutos de 
avaliação direta dos parâmetros. Pascoe et al. (1994), ao avaliarem os efeitos do halotano em cães submetidos à hipovolemia experimental, observaram que a função cardiovascular foi parcialmente recuperada após 30 minutos da indução da hemorragia. Entretanto, Kawana (1995) descreve que o sevofluorano produz queda da PAM, do DC, índice cardíaco, aumento da FC, assim como vasodilatação, reduzindo a RVS (Kersten et al., 1994).

Quanto à biotelemetria, observou-se que os animais que receberam somente solução $\mathrm{NaCl}$ $7,5 \%$ mantiveram a $\mathrm{FC}$ e as pressões arteriais no mesmo patamar dos animais tratados com solução $\mathrm{NaCl} 7,5 \%$ associada ao HES, ou seja, embora tenham sido observadas diferenças cardiovasculares importantes no período inicial de avaliação (até T180), a longo prazo, todos os tratamentos foram eficazes na manutenção das variáveis cardiovasculares. Estes dados confirmam os obtidos por Velasco et al. (1980) e Kramer (1986), que descreveram que a administração de solução $\mathrm{NaCl} 7,5 \%$ aumentou a PAM, o DC e corrigiu os distúrbios metabólicos, por período superior a seis horas.

A opção pela utilização do sistema de biotelemetria como método de avaliação dos parâmetros fisiológicos propiciou inúmeros benefícios frente aos métodos convencionais de monitoramento de PA e FC, principalmente pela eliminação da interferência da contenção física ou da necessidade de procedimento anestésico visando à monitoração continuada, da mesma forma como descrito por Amar (2002) e Miyazaki (2002). Armentano et al. (1990) afirmaram que os níveis de confiabilidade e fidelidade dos dados, conseguidos por essa técnica, em relação aos obtidos por mensuração direta, permitem a utilização de uma amostra populacional bem menor.

Observou-se a formação de seroma e reação inflamatória ao redor de uma das unidades de transmissão, próximo aos eletrodos de aço inoxidável e no local de implante do cateter no lúmen da artéria femoral. Essa complicação foi resolvida no período de dez dias, praticando-se a drenagem por meio de punção com agulha hipodérmica (16G), aplicando-se bandagem compressiva, antibiótico e anti-inflamatório. Truett e West (1995) constataram a formação de seroma em cinco de oito animais avaliados.
Segundo Ilback et al. (2002), a implantação da unidade de transmissão envolve procedimento cirúrgico invasivo sendo conduta pós-operatória aliviar a dor, o processo inflamatório e prevenir a formação de seroma.

A reinfusão do sangue de cada animal após as 24 horas do estudo visou à restauração do perfil hematimétrico dos animais, buscando minimizar o trauma e recuperação clínica em um curto intervalo de tempo.

\section{CONCLUSÕES}

A administração de HES 130/0,4 associado à solução $\mathrm{NaCl} 7,5 \%$ propiciou o restabelecimento imediato dos parâmetros cardiovasculares no paciente hipovolêmico; a administração isolada de solução $\mathrm{NaCl}$ 7,5\% não foi capaz de restaurar a PAM no período imediato; a administração de cetamina racêmica ou cetamina levógira produziu efeitos cardiovasculares similares e, por meio da biotelemetria, comprovou-se que todos os tratamentos foram capazes de manter estáveis as pressões arteriais e a frequência cardíaca por um período de até 24 horas.

\section{AGRADECIMENTOS}

À Fundação de Amparo à Pesquisa do Estado de São Paulo (FAPESP), pela concessão de bolsa de estudos e auxílio financeiro, processos 03/011119 e 03/13671-9.

\section{REFERÊNCIAS BIBLIOGRÁFICAS}

AMAR, D. The effects of advanced age on the incidence of supraventricular arrhythmias after pneumonectomy in dogs. Anesth. Analg., v.94, p.1132-1136, 2002.

ANDERSON, N.H. Telemetry for cardiovascular monitoring in a pharmacological study: New approaches to data analysis. Hypertension, v.33, p.248-255, 1999.

ARMENTANO, R. Telemetry of aortic pressure in unrestrained animals: validation of the method over a wide range of blood pressure (from 40 to $200 \mathrm{mmHg}$. Med. Prog. Tec., v.16, p.125-129, 1990.

COOK, D.J.; CARTON, E.G.; HOUSMANS, P.R. Mechanism of the positive inotropic effect of ketamine in isolated ferret ventricular 
papillary muscle. Anesthesiology, v.74, p.880888, 1991.

CROMHOUT, A. Ketamine: Its use in the emergency department. Emerg. Med., v.15, p.155-159, 2003.

DUQUE, J.C.M.; SOUZA, A.P.; NUNES, N. et al. Continuous infusion of ketamine in hypovolemic dogs anesthetized with desflurane. J. Vet. Emerg. Crit. Care, v.15, p.92-99, 2005.

HASKINS, S.C.; PASCOE, P.J.; ILKIW, J.E. et al. The effect of moderate hipovolemia on cardiopulmonary function in dogs. J. Vet. Emerg. Crit. Care, v.15, p.100-109, 2005.

ILBACK, N.G.; GUNNARSSON, K.; STALHANDSKE, T. Effects of rhIGF and insulin-unduced hypoglycemia on cardiovascular parameter recorded with telemetry in the conscious dog. Pharmacol. Toxicol., v.90, p.7381, 2002.

INGWERSEN, W.; ALLEN, D.G.; DYSON, D.H. et al. Cardiopulmonary effects of a ketamine/acepromazine combination in hypovolemic cats. Can. J. Vet. Res., v.52, p.423427, 1988.

KAWAMA, S. Comparação das alterações hemodinâmicas induzidas pelo sevofluorano e o halotano em pacientes pediátricos. Can. J. Anaesthesiol., v.42, p.603-607, 1995.

KERSTEN, J. Perfusão do miocárdio isquêmico durante a anestesia com sevofluorano. Anesthesiology, v.81, p.995-1004, 1994.

KONSAYREEPONG， S.; COOK， D.J.; HOUSMANS, P.R. Mechanisms of the direct, negative inotropic effect of ketamine in isolated ferret and frog ventricular myocardium. Anesthesiology, v.79, p.313-322, 1993.
KONRAD, C.J.; MARKL, T.J.; SCHUEPFER, G.K. In vitro effects of different medium molecular hydroxyethyl starch solutions and lactated ringer's solution on coagulation using sonoclot for analysis. Anesth. Analg., v.90, p.274-279, 2000.

KRAMER, G.C. Small volume ressuscitation with hypertonic saline dextram solution. Surgery, v.100, p.239-246, 1986.

KREIMEIER, U.; THIEL, M.; PETER, K. et al. Small-volume hyperosmolar resuscitation. Acta Anaesthesiol. Scand., v.41, p.302-306, 1997.

MIYAZAKI, H. Ranges of diurnal variation and the pattern of body temperature, blood pressure and heart rate in laboratory beagle dogs. Exp. Anim., v.1, p.95-98, 2002.

NAM, T.C. Studies on the effect of ketamine hydrochloride on dogs after bleeding. I. Clinical findings and acid-base balance. Jpn. J. Vet. Sci., v.39, p.273-281, 1977.

PASCOE, P.J.; HASKINS, S.C.; ILKIW, J.E. et al. Cardiopulmonary effects of halothane in hypovolemic dogs. Am. J. Vet. Res., v.55, p.121126, 1994.

TAN, T.S.; TAN, K.H.; NG, H.P. et al., The effects of hypertonic saline solution $(7.5 \%)$ on coagulation and fibrinolysis: an in vitro assessment using thromboelasography. Anaesthesia, v.57, p.644-648, 2002.

TRUETT, A.A.; WEST, D.B. Validation of a radiotelemetry system for continuous blood pressure and heart rate monitoring in dogs. Lab. Anim. Sci., v.45, p.299-302, 1995.

VELASCO, I.T.; PONTIERI, V.; ROCHA E SILVA, M. et al. Hypertonic $\mathrm{NaCl}$ and severe hemorrhagic shock. Am. J. Physiol., v.239, p.664-673, 1980. 